\title{
An unanticipated lack of consensus cytotoxic $T$ lymphocyte epitopes in HIV-1 databases: the contribution of prediction programs
}

\author{
Ingrid M.M. Schellens ${ }^{a}$, Can Keşmir ${ }^{b}$, Frank Miedema ${ }^{a}$, \\ Debbie van Baarle ${ }^{a}$ and José A.M. Borghans ${ }^{a, b}$
}

\begin{abstract}
Background: Most consensus HIV-1-specific cytotoxic T lymphocytes epitopes presented via intensively studied HLA molecules are thought to be known

Objective: To identify possible novel HIV-1 epitopes for HLA-B27 and HLA-B57; two HLA types which are abundantly studied because of their correlation with slow HIV disease progression.
\end{abstract}

Methods: HIV-1 consensus subtype B sequences were analysed using peptide prediction programs based on major histocompatibility complex binding, proteasomal cleavage and TAP (transporter associated with antigen processing) transport. Recognition of the novel identified epitopes by cytotoxic T lymphocytes was tested using interferon- $\gamma$ ELISpot assay.

Results: In total, 22 novel epitopes predicted to be presented by either HLA-B27 or HLA-B57 were selected. Of these, $86 \%$ elicited significant immune responses in HIV-1infected individuals.

Conclusions: These data show that numerous HIV-1 epitopes remain to be identified, and that prediction programs are powerful tools for this purpose.

(c) 2008 Wolters Kluwer Health | Lippincott Williams \& Wilkins

AIDS 2008, 22:33-37

Keywords: CTL epitopes, HIV-1, HLA-B27, HLA-B57, peptide prediction programs

\section{Introduction}

There is increasing evidence that cytotoxic T lymphocytes (CTL) play an important role in the control of HIV infection [1-4]. Studies addressing the role of HIV-1specific CTL responses are, however, complicated by the large population diversity of human leukocyte antigen (HLA) molecules. Nevertheless, intense research has identified a large variety of HIV-1 peptides presented by different HLA molecules that are known to elicit immune responses in HIV-1-infected individuals. Most of these peptides were identified after stimulation of cells with overlapping peptide pools, followed by the identification of the optimal epitope and the HLA-restriction element. It is commonly believed that most consensus HIV-1- specific CTL epitopes that are presented via intensively studied HLA molecules are now known. To examine whether HIV-1 databases are indeed nearly complete, the current study sought to identify novel HIV-1 epitopes presented by HLA-B27 or HLA-B57, two HLA alleles that are frequently studied because of their well-known correlation with slow HIV-1 disease progression $[5,6]$.

\section{Methods}

\section{Peptide predictions}

Peptide prediction programs based on major histocompatibility complex (MHC) binding [7,8], proteasomal cleavage [9] and TAP (transporter associated with antigen

From the ${ }^{a}$ Department of Immunology, University Medical Center, and the ${ }^{\mathrm{b}}$ Theoretical Biology/Bioinformatics, Utrecht University, Utrecht, the Netherlands.

Correspondence to Dr J.A.M. Borghans, University Medical Center Utrecht, KC02.085.2, Lundlaan 6, 3584 EA Utrecht, the Netherlands.

E-mail: j.borghans@umcutrecht.nl

Received: 24 July 2007; revised: 13 August 2007; accepted: 17 August 2007. 
processing) transport [10] (www.cbs.dtu.dk/services) were applied to selected novel epitopes predicted to be presented via either HLA-B27 or HLA-B57. Peptides were only selected if they fulfilled the following criteria: (i) novelty, that is, not reported in the Los Alamos HIV database [11]; (ii) a predicted proteasomal cleavage score $>0.5$ [9]; (iii) a predicted TAP transport score $>1$ [10]; and (iv) a normalized [12] HLA-binding affinity $>1.5$, which corresponds to good binders within the top $1 \%$ for each HLA allele.

\section{Measurement of epitope recognition by cytotoxic T lymphocytes}

Cryopreserved peripheral blood mononuclear cells (PBMC) were thawed and incubated in triplicate at a final concentration of $10^{5}$ cells/well in the presence or absence of $20 \mu \mathrm{g} / \mathrm{ml}$ peptide for $20-24 \mathrm{~h}$ at $37^{\circ} \mathrm{C}$. The capacity of PBMC to produce interferon (IFN) $-\gamma$ was measured by an ELISpot assay. Phytohemagglutinin (Murex, Dartford, UK) stimulation served as a positive control to test the capacity of PBMC to produce interferon (IFN) $\boldsymbol{\gamma}$, and medium alone served as a negative control. Samples with $>100$ spot-forming units (SFU) per $10^{6}$ PBMC after subtraction of the negative control value were considered positive.

\section{Statistical analysis}

Data were analysed using SPSS 12.01 software (SPSS, Chicago, Illinois, USA). Differences between predicted and known epitopes were analysed using Mann-Whitney or $\chi^{2}$ tests. $P \leq 0.05$ was considered statistically significant.

\section{Results}

\section{Strong cytotoxic T lymphocyte responses towards peptides predicted to be presented through HLA-B27 or HLA-B57}

PBMC were collected from HIV-infected individuals from the Amsterdam Cohort Studies on HIV-1 infection and AIDS. In total, 22 novel HIV-1 epitopes (Table 1) were selected for which recognition by CTL was tested in PBMC from a mean of nine (range, 8-9) individuals carrying the HLA-B27 allele or six (range, 5-8) individuals carrying the $H L A-B 57$ allele, as previously described $[13,14]$. Peptide prediction scores of the novel epitopes were comparable to prediction scores obtained for peptides already known to bind to HLA-B27 or HLAB57 listed in the Los Alamos HIV database (MannWhitney, $P=0.12$ ).

All 11 peptides predicted to be presented through HLAB27 induced IFN- $\gamma$ production in at least one HLA-B27positive individual [a positive IFN- $\gamma$ response in a median of $22 \%$ of tested individuals (range, 11-89)]. This was also the case for 8 out of the 11 epitopes predicted to be presented through HLA-B57 (a positive IFN- $\gamma$ response in a median $20 \%$ of tested individuals (range, $0-63$ )]. In contrast, these peptides did not induce IFN- $\gamma$ production in five HIV-negative donors expressing HLA-B27 or in five donors expressing HLA-B57, indicating that the observed responses are indeed HIV specific (data not shown). Overall, the magnitude of CTL responses towards the predicted peptides was similar to the responses towards known HLA-B27- or HLA-B57restricted peptides from the Los Alamos HIV database (Table 1 gives peptide characteristics) analysed in the same HIV-1-infected individuals (Fig. 1). Only two HLAB27-restricted peptides (TR9, EF9) and one HLA-B57restricted peptide (DW9) induced significantly lower responses, while one HLA-B27-restricted peptide (KY9) even induced a significantly higher response (MannWhitney, $P<0.01)$. The latter peptide was recognized by as many as eight out of nine individuals tested $\left(\chi^{2}\right.$ test comparison with the known HLA-B27-restricted peptides: $P=0.03$ ). None of the predicted peptides induced a positive IFN- $\gamma$ response in HIV-1-infected individuals who did not express HLA-B27 or HLA-B57 (data not shown).

Although the magnitude of the CTL response against the newly identified peptides was similar to the responses against known HIV-1 peptides from the Los Alamos HIV database, the number of individuals responding to the different predicted peptides was somewhat lower. Of the 11 HLA-B27-restricted peptides, five induced responses in a fraction of individuals comparable to (or in the case of KY9 even higher than) known peptides from the Los Alamos HIV database. Also five of the eight HLA-B57 peptides were recognized by a similar fraction of individuals compared with known HLA-B57 restricted peptides.

\section{Discussion}

After more than 20 years of intensive research, it is believed that most consensus HIV-specific CTL epitopes are known. Unexpectedly, we found that there are still numerous epitopes to be discovered. Using peptide prediction programs, we identified 22 novel epitopes presented via either HLA-B27 or HLA-B57 which could induce strong CTL responses. A possible explanation for the lower fraction of individuals responding to certain predicted epitopes is that only two-digit HLA typing of the individuals participating in this study was available. Since the peptide predictions were performed using the most abundant four-digit HLA type of each HLA serotype, lack of response to certain predicted HLA epitopes may result from absence of the actual fourdigit HLA type. The lower number of individuals responding to certain predicted peptides could also reflect higher sequence variability within these epitopes, as all three HLA-B57-restricted peptides and four of the six 
Table 1. Characteristics of the HLA-B27- and HLA-B57-restricted cytotoxic T lymphocyte epitopes studied.

\begin{tabular}{|c|c|c|c|c|}
\hline Source & Epitope & Sequence & Gene & Recognition (\%) \\
\hline \multicolumn{5}{|c|}{ HLA-B27-restricted epitopes } \\
\hline \multirow[t]{11}{*}{ Predicted } & TR9 & TRANSPTRR & pol & 22 \\
\hline & KY9 & KRKGGIGGY & pol & 89 \\
\hline & IY9 & IRDKVQKEY & env & 22 \\
\hline & SL9 & SRAKWNNTL & env & 22 \\
\hline & AY9 & ARVLAVERY & env & 33 \\
\hline & EF9 & EREVLVWKF & nef & 11 \\
\hline & AW9 & ARRNRRRRW & rev & 22 \\
\hline & NW9 & NRWQVMIVW & vif & 25 \\
\hline & SY9 & SRKAKGWFY & vif & 22 \\
\hline & EL9 & ERFAVNPGL & gag & 22 \\
\hline & AW9 & ARLVITTYW & vif & 44 \\
\hline \multirow[t]{7}{*}{ Los Alamos } & RL8 & RYLKDQQL & env & 44 \\
\hline & GK9 & GRRGWEALK & env & 88 \\
\hline & RI10 & RRQDILDLWI & nef & 44 \\
\hline & RW8 & RYPLTFGW & nef & 44 \\
\hline & KK10 & KIRLRPGGKK & gag & 11 \\
\hline & KK10 & KRWIILGLNK & gag & 100 \\
\hline \multirow{2}{*}{\multicolumn{5}{|c|}{ HLA-B57-restricted epitopes }} \\
\hline & & & & \\
\hline \multirow[t]{11}{*}{ Predicted } & KW9 & KNYQHLWRW & env & 0 \\
\hline & TW9 $^{\mathrm{a}}$ & TTQLFNSTW & env & 0 \\
\hline & SW9 & STWNVNGTW & env & 17 \\
\hline & $\mathrm{DW}^{\mathrm{a}}$ & DPEREVLVW & nef & 0 \\
\hline & VW9 & VTKLTEDRW & vif & 60 \\
\hline & TW9 & TINEEAAEW & gag & 20 \\
\hline & PW9 & PTRRELQVW & pol & 25 \\
\hline & FW9 & FSFPQITLW & pol & 25 \\
\hline & KW9 & KLPIQKETW & pol & 38 \\
\hline & PW9 & PVDPRLEPW & tat & 13 \\
\hline & IW9 & IATESIVIW & pol & 63 \\
\hline \multirow[t]{14}{*}{ Los Alamos } & GK9 & GRRGWEALK & env & 22 \\
\hline & AW9 & AVRHFPRIW & $v p r$ & 25 \\
\hline & GY14 ${ }^{\mathrm{a}}$ & GPGVRYPLTFGWCY & nef & 0 \\
\hline & GP10 & GQMVHQAISP & gag & 29 \\
\hline & NF20 & NAWVKVVEEKAFSPEVIPMF & gag & 25 \\
\hline & IF9 & ISKKAKGWF & vif & 25 \\
\hline & YT9 & YFPDWQNYT & nef & 38 \\
\hline & IW9 & ISPRTLNÄW & gag & 44 \\
\hline & QW9 & QASQEVKNW & gag & 67 \\
\hline & KY11 & KAVRRLIKFLY & rev & 63 \\
\hline & HQ10 & HTQGYFPDWQ & nef & 67 \\
\hline & IW9 & IVLPEKDSW & $r t$ & 67 \\
\hline & KF11 & KAFSPEVIPMF & gag & 67 \\
\hline & TW10 & TSTLQEQIGW & gag & 78 \\
\hline
\end{tabular}

${ }^{a}$ Epitopes that failed to elicit interferon- $\gamma$ responses of $>100$ spot-forming units $/ 10^{6}$ peripheral blood mononuclear cells after subtraction of the negative control value in HIV-1-infected individuals.

HLA-B27-restricted peptides recognized in fewer individuals had a significantly higher entropy score compared with the other HLA-B57- or HLA-B27-restricted peptides $\left(\chi^{2}\right.$ test, $\left.P<0.05\right)$. A similar negative correlation between the entropy score of a peptide and the number of individuals responding to the peptide has been described previously [15]. In the present study, the selection of potential novel CTL epitopes was based on peptide prediction programs for MHC binding, TAP transport and proteasomal cleavage. Recently, Assarsson et al. [16] have shown that binding to MHC molecules is the most restricting step influencing immunodominance, although other factors including $\mathrm{T}$ cell receptor repertoire and immunoregulatory mechanisms also play a significant role. Interindividual differences in the last two factors may also have contributed to the observation that some individuals did while others did not respond to our novel identified epitopes.

Since we aimed to detect novel epitopes that could be recognized by a large number of HIV-1-infected individuals, all peptide predictions were performed using HIV-1 consensus subtype B sequences. The fact that we found a significant physiological CTL response in HIV-1infected individuals against as much as $86 \%$ of the novel predicted epitopes suggests that many consensus HIV epitopes are still missing from the HIV-1 epitope databases despite the intensity of research based on overlapping HIV peptide pools. In line with this, Betts et al. [17] found a significantly broader CTL response after 
(a)

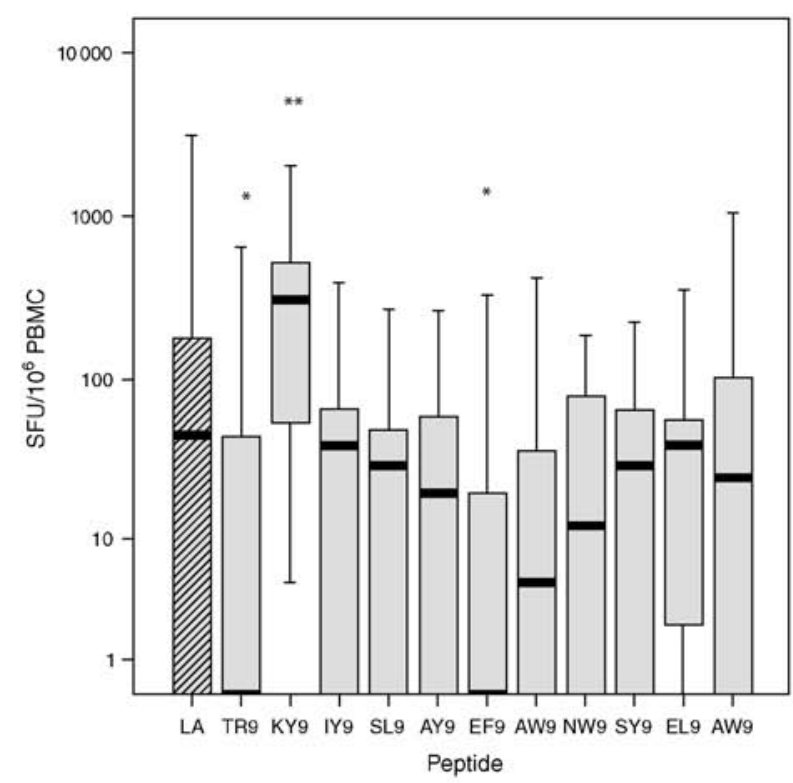

(b)

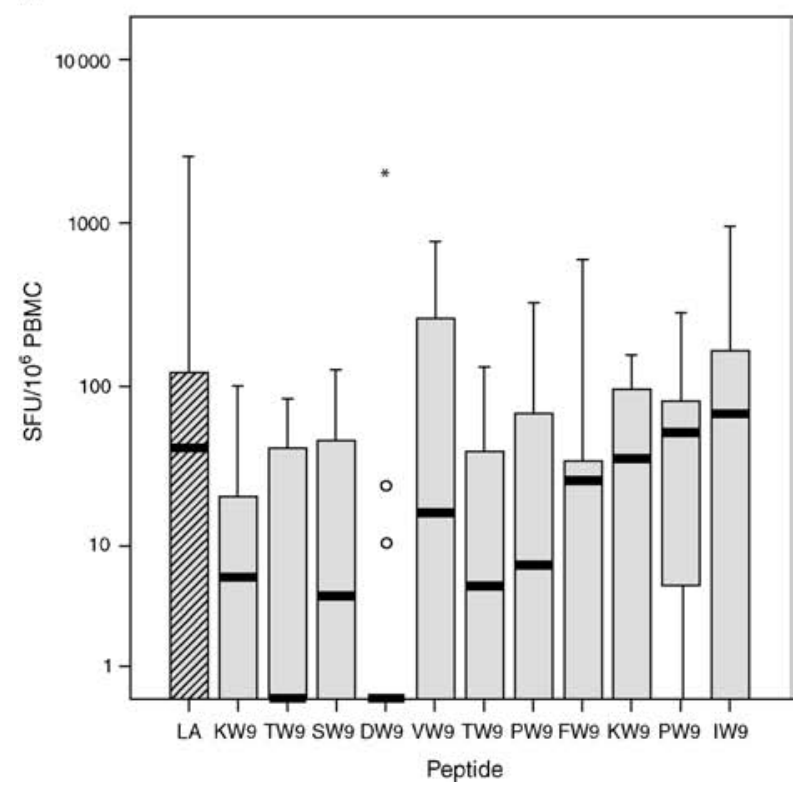

Fig. 1. Magnitude of cytotoxic $T$ lymphocyte responses towards peptides predicted to be presented through HLAB27 or HLA-B57. The responses towards peptides predicted to be presented through HLA-B27 (a) or HLA-B57 (b) were compared with responses towards 7 or 14 peptides described to be presented via these HLA proteins, respectively, in the Los Alamos database (LA). Interferon- $\gamma$ responses were measured as number of spot-forming units (SFU) $/ 10^{6}$ peripheral blood mononuclear cells (PBMC) after subtraction of the medium value. The different predicted peptides are shown on the $x$ axis. Significant differences compared with the peptides from the Los Alamos database were calculated using the Mann-Whitney test; one asterisk indicates that the magnitude of response after stimulation with the peptide was significantly lower compared with that of the database peptides and two asterisks indicates that the response was significantly higher. stimulation with overlapping peptide pools covering the whole HIV genome than with a pool of optimized peptides. In addition, Liu et al. [18] have recently studied an HIV-1-infected individual for 3 years after acute infection and have shown that 14 of the 25 recognized epitopes had not been reported previously in HIV-1 databases. As shown by Altfeld et al. [15], the use of peptides based on autologous HIV sequences instead of consensus sequences significantly enhances the detection of CTL responses, especially against the more variable regions of HIV. Our study clearly shows that prediction programs provide powerful tools to help the identification of such autologous HIV epitopes.

In the current study, we detected CTL responses by measuring the production of only one cytokine, IFN- $\gamma$, which is the last cytokine to be lost during chronic antigen stimulation [19] and which is widely used as a means of identifying specific CTL. It was recently shown that the presence of polyfunctional $\mathrm{T}$ cells, which are capable of producing multiple cytokines and chemokines and are able to proliferate, is associated with virus control [20]. Further research focusing on the polyfunctionality of the CTL responses induced by these newly identified epitopes, but also by previously identified HIV epitopes, could reveal information about the relative importance of these CTL responses in virus control.

\section{Acknowledgements}

Sponsorship: This work was financially supported by a grant from the Landsteiner Foundation for Blood Transfusion Research (grant 0317). The Amsterdam Cohort Studies on HIV-1 Infection and AIDS (a collaboration between the Amsterdam Health Service, the Academic Medical Center of the University of Amsterdam, Sanquin Blood Supply Foundation and the University Medical Center Utrecht) are part of the Netherlands HIV-1 Monitoring Foundation and financially supported by the Netherlands National Institute for Public Health and the Environment.

\section{References}

1. Goulder PJ, Watkins DI. HIV and SIV CTL escape: implications for vaccine design. Nat Rev Immunol 2004; 4:630-640.

2. Schmitz JE, Kuroda MJ, Santra S, Sasseville VG, Simon MA, Lifton MA, et al. Control of viremia in simian immunodeficiency virus infection by CD8+ lymphocytes. Science 1999; 283:857860.

3. Jin X, Bauer DE, Tuttleton SE, Lewin S, Gettie A, Blanchard J, et al. Dramatic rise in plasma viremia after $\mathbf{C D 8}(+) \mathbf{T}$ cell depletion in simian immunodeficiency virus-infected macaques. J Exp Med 1999; 189:991-998.

4. Borrow P, Lewicki H, Hahn BH, Shaw GM, Oldstone MB. Virusspecific CD8 + cytotoxic T-lymphocyte activity associated with control of viremia in primary human immunodeficiency virus type 1 infection. / Virol 1994; 68:6103-6110. 
5. Kaslow RA, Carrington M, Apple R, Park L, Munoz A, Saah AJ, et al. Influence of combinations of human major histocompatibility complex genes on the course of HIV-1 infection. Nat Med 1996; 2:405-411.

6. Migueles SA, Sabbaghian MS, Shupert WL, Bettinotti MP, Marincola FM, Martino L, et al. HLA B $* 5701$ is highly associated with restriction of virus replication in a subgroup of HIV-infected long term nonprogressors. Proc Natl Acad Sci USA 2000; 97:2709-2714.

7. Nielsen M, Lundegaard C, Worning P, Lauemoller SL, Lamberth $\mathrm{K}$, Buus $\mathrm{S}$, et al. Reliable prediction of T-cell epitopes using neural networks with novel sequence representations. Prot Sci 2003; 12:1007-1017.

8. Buus S, Lauemoller SL, Worning P, Kesmir C, Frimurer T, Corbet $\mathrm{S}$, et al. Sensitive quantitative predictions of peptide-MHC binding by a 'Query by Committee' artificial neural network approach. Tissue Antigens 2003; 62:378384.

9. Kesmir C, Nussbaum AK, Schild H, Detours V, Brunak S. Prediction of proteasome cleavage motifs by neural networks. Prot Eng 2002; 15:287-296.

10. Peters B, Bulik S, Tampe R, van Endert PM, Holzhutter HG. Identifying MHC class I epitopes by predicting the TAP transport efficiency of epitope precursors. I Immunol 2003; 171:1741-1749.

11. Korber BTM, Brander C, Haynes BF, Koup R, Moore JP, Walker BD, et al. HIV Molecular Immunology 2005. Los Alamos, NM: Los Alamos National Laboratory of Theoretical Biology and Biophysics; 2006 (LA-UR 06-0036).

12. Sturniolo T, Bono E, Ding J, Raddrizzani L, Tuereci O, Sahin U, et al. Generation of tissue-specific and promiscuous HLA ligand databases using DNA microarrays and virtual HLA class II matrices. Nat Biotechnol 1999; 17:555-561.
13. van Baarle D, Hovenkamp E, Callan MF, Wolthers KC, Kostense $\mathrm{S}$, Tan LC, et al. Dysfunctional Epstein-Barr virus (EBV)-specific CD8(+) T lymphocytes and increased EBV load in HIV-1 infected individuals progressing to AIDS-related non-Hodgkin lymphoma. Blood 2001; 98:146-155.

14. Lalvani A, Brookes R, Hambleton S, Britton WJ, Hill AV, McMichael AJ. Rapid effector function in CD8+ memory $T$ cells. J Exp Med 1997; 186:859-865.

15. Altfeld M, Addo MM, Shankarappa R, Lee PK, Allen TM, Yu XG, et al. Enhanced detection of human immunodeficiency virus type 1-specific T-cell responses to highly variable regions by using peptides based on autologous virus sequences. I Virol 2003: 77:7330-7340.

16. Assarsson E, Sidney J, Oseroff C, Pasquetto V, Bui HH, Frahm N, et al. A quantitative analysis of the variables affecting the repertoire of $T$ cell specificities recognized after vaccinia virus infection. I Immunol 2007; 178:7890-7901.

17. Betts MR, Ambrozak DR, Douek DC, Bonhoeffer S, Brenchley JM, Casazza JP, et al. Analysis of total human immunodeficiency virus (HIV)-specific CD4(+) and CD8(+) T-cell responses: relationship to viral load in untreated HIV infection. / Virol 2001; 75:11983-11991.

18. Liu Y, McNevin J, Cao J, Zhao H, Genowati I, Wong K, et al. Selection on the human immunodeficiency virus type 1 proteome following primary infection. / Virol 2006; 80:9519-9529.

19. Wherry EJ, Blattman JN, Murali-Krishna K, van der Most R, Ahmed R. Viral persistence alters CD8 T-cell immunodominance and tissue distribution and results in distinct stages of functional impairment. J Virol 2003; 77:4911-4927.

20. Betts MR, Nason MC, West SM, De Rosa SC, Migueles SA, Abraham J, et al. HIV nonprogressors preferentially maintain highly functional HIV-specific CD8+ T cells. Blood 2006; 107:4781-4789. 